\title{
Study on Comprehensive Evaluation Method for Track Irregularity Based on HSMM
}

\author{
Limei GUO',a, HaiXin Lin²,b, Xianghua WU ${ }^{3, c}$, Hanyu Cui ${ }^{4, d}$ \\ 1,2,3,4 Central South University,Hunan, Changsha, 410083, China \\ axdjh2007@163.com, ${ }^{\text {b314683714@ qq.com, }{ }^{\mathrm{c}} 78251530 @ q q . c o m,{ }^{\mathrm{d}} 715711942 @ q q . c o m}$
}

\begin{abstract}
Keywords: Track irregularity, hidden semi-Markov models, track quality index, multi-source track detection data.

Abstract: Evaluation of track irregularity is the foundation of track maintenance and traffic safety. A comprehensive evaluation model based on hidden semi-Markov models was proposed to evaluate track irregularity in a certain length, which used the multiple dynamic and static detection data. The model also took into account the different affection of track structure, passed total weight and allowed velocity. The features of track quality index, track complex irregularity, acceleration amplitude were extracted to train the proposed model. Finally, the effectiveness of the proposed model is verified by the simulation results.
\end{abstract}

\section{Introduction}

Track irregularity is the deviation of track geometry and spatial location, including the track vertical irregularities, alignment irregularities, gauge, crosslevel irregularity, and warp. It is very important to master the track irregularity and its change law timely, which is helpful to schedule track maintenance scientifically and ensure the safety of the track working. Both domestic and foreign scholars in recent years have done a lot of research on evaluating track irregularity.

In [1], a qualitative and quantitative investigation on the judgment method of track irregularity was made based on the track irregularity power spectrum density of railway. A comprehensive evaluation system based on RAMS is proposed in [2], which analyses the track irregularity states by combining the characteristic quantity of track irregularities and vehicle dynamic response. Lecture [3] compares and analyzes the dynamic responses aroused by speed of the train and amplitude of the complex irregularities, and provides theoretical safety criterion for railway maintenance. In [4], The transferring relationship between the long-wave profile irregularity and the car-body vertical acceleration is described using nonparametric model and ARX model.

Now, there are still some deficiencies on evaluate track irregularities. First, with the development of modern detection technology, track inspection car, track geometry inspection instrument, train's vibration analyzer and dynamic inspection car are used to obtain the multiple track state parameters from different perspectives. But now the multi-source track detection data are not integrated effectively. Second, track irregularity is influenced by many factors, such as track structure, passed total weight and allowed velocity. Therefore, the evaluation model must consider all the factors.

In this paper, we study the combined effects of track structure, passed total weight and allowed velocity to evaluate the track irregularity based on multi-source track detection data. A comprehensive evaluation model based on hidden semi-Markov models(HSMM) is proposed to valuate track irregularity in a certain length. HSMM has strong modeling capability as well as good analytical ability, it is an extension of the popular hidden Markov model(HMM), except each state can emit a sequence of observations. This makes it suitable for evaluating track irregularity.

\section{The Comprehensive Evaluation Model for Track State}

Now, many different methods are used to evaluate the track state: scoring method(or peak value management), the average characteristics of track irregularities (such as track quality index(TQI)) 
method, generalized energy index (GEI) method and power spectrum density method. Literature [5] said that scoring method is difficult to reflect the track's actual condition objectively, and there is not detail criteria for GEI method and power spectrum density method in China. So, in this paper, we use the average characteristics of track irregularities method to evaluate track irregularity comprehensively. The proposed model is showed in Figure 1.

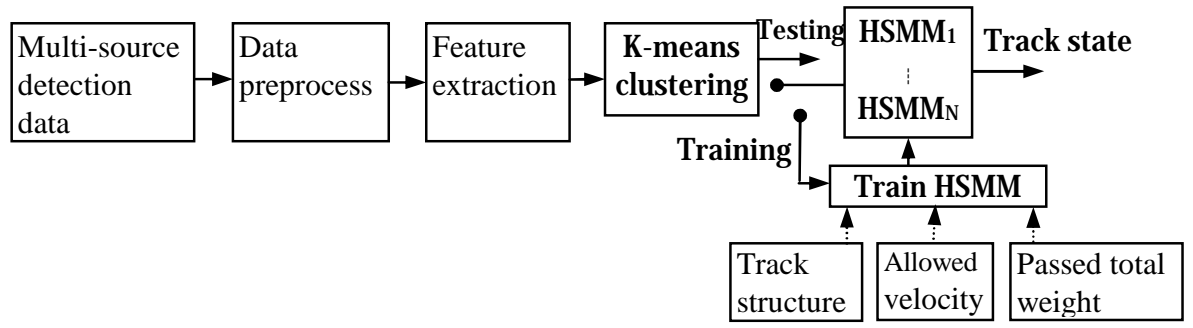

Fig.1 A comprehensive evaluation model for track irregularity

This model is comprehensively considered the impacts of track structure, passed total weight and allowed velocity, different track has different model. Data preprocess is to deal with the singularities, eliminate noise and false data. When HSMM model is used to evaluate the track states, the task is to recognize $\mathrm{N}$ different states of track irregularity, it includes two procedures. First, $\mathrm{N}$ different HSMMs should be trained to get the HSMM model parameters. Second, for the procedure of evaluation, the observation sequence is inputted to each of the $\mathrm{N}$ trained HSMMs respectively, the HSMM with highest log-likelihood output is presented the state of the track.

\section{HSMM}

HSMM allows the underlying process to be a semi-Markov chain with a variable duration or sojourn time for each state. Unlike HMM, in HSMMs, the probability of transition from state $j$ to state $i$ at time $t$ depends on the duration spent in state $j$ prior to time $t$. We assume $N$ is the state number, $M$ is the observation number in state $i$, the characteristic of HSMM can be describe as

$$
\lambda=(\mathrm{N}, \mathrm{M}, \pi, \mathrm{A}, \mathrm{D}, \mathrm{B})
$$

Where $\pi$ is the initial probability distribution $\pi=\left\{\pi_{1}, \pi_{2}, \ldots, \pi_{\mathrm{N}}\right\}$

$$
\pi_{i}=\mathrm{P}\left(S_{1}=i\right) \geq 0,1 \leq i \leq N \text {, with } \sum_{i=1}^{N} \pi_{i}=1
$$

$A$ is the state transition probability matrix $A=\left\{a_{i j}(\mathrm{~d})\right\}_{N \times N}$

$$
\begin{aligned}
& a_{i j}(d)=P\left(q_{t+1}=j \mid q_{t}=i, d_{t}(i)=d\right) \quad 1 \leq i, j \leq N, 1 \leq d \leq D_{i} \\
& \sum_{j=1}^{N} a_{i j}(d)=1 \quad 1 \leq i \leq N, 1 \leq d \leq D_{i}
\end{aligned}
$$

where $D_{i}$ is max staying time in state $i . B$ is the observed matrix $B=\left\{b_{j}(k)\right\}_{N \times M}$

$$
b_{j}(k)=P\left(v_{k} \mid s_{t}=i\right) \quad 1 \leq k \leq M, 1 \leq i \leq N
$$

where $v_{i}=\left\{v_{1}, v_{2}, \ldots, v_{\mathrm{M}}\right\}$ is the observation symbols in state $i . D$ is the sojourn time distribution(denoted by $\left.D\left(P_{i}(d)\right)(i=1,2, \ldots, N)\right)$. The detailed discussion on HSMM algorithm can be found in [6].

\section{Feature extraction}

The multi-source track detection data come from dynamic inspection car, track inspection car, track inspection instrument, train's vibration analyzer, and train-mounted line tester. Here we extract TQI, track complex irregularity and acceleration index as the features to evaluate the track irregularity.

1). Track quality index(TQI)

TQI is an important index to evaluate the quality of railway track, it is the summation of standard deviations of seven irregularities, that is vertical irregularities (left and right), alignment irregularities (left and right), gauge, crosslevel irregularity, and warp, in each $200 \mathrm{~m}$ long track section 


$$
\begin{gathered}
T Q I=\sum_{i=1}^{7} \sigma_{i} \\
\sigma_{i}=\sqrt{\frac{1}{n} \sum_{j=1}^{n}\left(x_{i j}-\bar{x}_{i}\right)^{2}}, \bar{x}_{i}=\frac{1}{n} \sum_{j=1}^{n} x_{i j}
\end{gathered}
$$

Where $\mathrm{N}(\mathrm{N}=7)$ denotes irregularity number, $\sigma_{i}$ is standard deviation of each irregularity, $x_{i j}$ is the value of local irregularities, $\bar{x}_{i}$ is average value deviation, $n$ is the number of sampling points.

2). Track complex irregularity (TCI)

TCI come from a combination of two or more individual track irregularities. Because the impacts of alignment and cross level track complex irregularities is serious ${ }^{[3]}$. In this paper, the equation of alignment and cross level track complex irregularities is given by

$$
z_{j}=\left|x_{1 j}-\zeta x_{2 j}\right|
$$

where $x_{1 j}$ is value of alignment irregularity, $x_{2 j}$ is value of cross level irregularity, $\zeta$ is weighting factor. In practice, standard deviation is used to represent the track complex irregularity

$$
R=\sqrt{\frac{1}{n} \sum_{j=0}^{n} z_{j}^{2}-\bar{z}_{j}^{2}}
$$

where, $\bar{z}$ is the average value of complex irregularity, $\bar{z}=\frac{1}{n} \sum_{j=1}^{n} z_{j}, n$ is the number of sampling. It is important to choose the weight factor between track alignment irregularity and level irregularity in the management of track complex irregularities, literature [15] discussed the safety limit value of complex irregularity for heavy haul railway.

3). Acceleration index (AI)

Acceleration includes the vertical vibration acceleration of the car body and the lateral vibration acceleration of the car body, $\mathrm{AI}$ is given by

$$
\begin{gathered}
A I=\alpha_{v} \sigma_{v}+\alpha_{H} \sigma_{H} \\
\sigma_{v}=\sqrt{\frac{1}{n} \sum_{j=1}^{n}\left(x_{v j}-\bar{x}_{v}\right)^{2}}, \quad \sigma_{H}=\sqrt{\frac{1}{n} \sum_{j=1}^{n}\left(x_{H j}-\bar{x}_{H}\right)^{2}}, \bar{x}_{v}=\frac{1}{n} \sum_{j=1}^{n} x_{v j}, \quad \bar{x}_{H}=\frac{1}{n} \sum_{j=1}^{n} x_{H j}
\end{gathered}
$$

wher $\sigma_{v}$ is the standard deviation of vertical vibration acceleration, $\sigma_{H}$ is the standard deviation of lateral vibration acceleration, $\overline{x_{v}}$ is the average value of $x_{v j}, \overline{x_{H}}$ is the average value of $x_{H j}, \alpha_{v}, \alpha_{H}$ are weighting factors for the standard deviation of vertical vibration acceleration and the standard deviation of lateral vibration acceleration respectively.

\section{Simulation Results}

In order to verify the validity of the proposed model, computer experiments are employed. The data were divided into two data subsets, say training data and testing data, the state of track will be classified into three grades by their different value of track irregularity: good, middle and worse. Good represents that the track irregularity does not exceed the pre-defined management threshold, middle and worse represent that the track irregularity exceed $10 \%$ and $20 \%$ of pre-defined management threshold respectively.

Features extracted from the multi-source track detection data include acceleration index and TQI of dynamic inspection car, acceleration index of track inspection car, TQI of track inspection instrument, acceleration index of train's vibration analyzer, acceleration index of train-mounted line tester, track complex irregularity of track inspection car and dynamic inspection car respectively.

Figure 2 illustrates the classification results for the testing data, Figure 2(a) is the result of track in good condition, which shows the testing samples of good condition have the highest log-likelihood. Figure 2(b) is the result of track in worse condition in another track line, which shows the testing samples of worse condition have the highest log-likelihood for the trained HSMM. All the sample data have been identified correctly. 


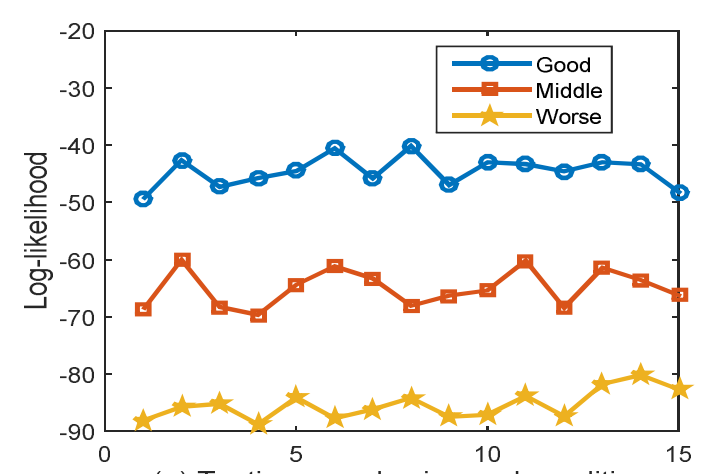

(a) Testing samples in good condition

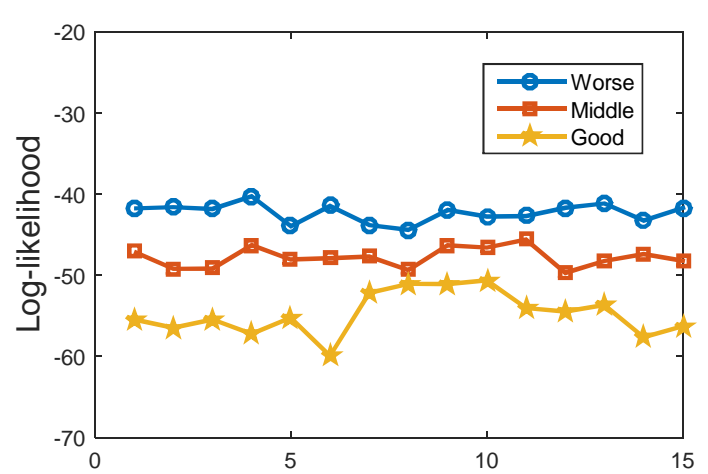

(b) Testing samples in worse condition

Fig.2. Output probability of HSMMs

\section{Conclusions}

Currently, various track inspection data store, analysis and utilize separately, which severely restrict the administrator to grasp the track states accurately and comprehensively and to schedule track maintenance scientifically. In this paper, a HSMM-based evaluation model are proposed to evaluate track states using multi-source track detection data, the model takes into account mutually complementary relationship between dynamic detection data and static detection data, The proposed comprehensive evaluation model will provide more scientific foundations and management references for track maintenance.

\section{Acknowledgements}

This work was financially supported by the China Railway Corporation (No.2014G009-F, No.2014G002-A)

\section{References}

[1] Xianmai Chen, Fengchun Yand, Xuesong CHAI, et al. Study on Evaluation Method of Power Spectrum Density (PSD) for the Qinhuangdao Shenyang Passenger Dedicated Railway Line. Journal of the China Railway Society, Vol.28,No. 4,( 2006),p. 84-88. (in Chinese)

[2] Xiong Kand, Weidong Wang, Jinzhao Liu. Research on Comprehensive Evaluation System for Track Irregularity of High-Speed Railway Based on RAMS. China Railway Science, Vol.34,No.2, (2013):13-17. (in Chinese)

[3] Ruiying Chen, Yude Xu, Jianping Cheng. Simulation and Safety Criterion on Complex Geometry Irregularities of Heavy Haul Railway. Journal of East China Jiaotong University, Vol.31,No.4, (2014), p.33-37

[4] Liubin Niu, Jinzhao Liu, Weidong WANG. Research on Relationship Model between Long-wave Profile Irregularity and Car-body Vertical Acceleration. Journal of the China Railway Society, Vol.37,No.4,( 2015),p.75-79. (in Chinese)

[5] Yigui Zhang. Discussion on the method of track quality evaluation. Shanghai Railway Science \& Technology, Vol.4,(2006), p.21-22. (in Chinese)

[6] Yu S.Z. Hidden semi-Markov models. Artificial Intelligence,Vol.174,No.2,(2010),p. 215-243 